\title{
Resonant isotropic optical magnetism of plasmonic nanoclusters in visible light
}

\author{
V. Ponsinet, ${ }^{1}$ P. Barois, ${ }^{1}$ S. M. Gali, ${ }^{1}$ P. Richetti, ${ }^{1}$ J. B. Salmon, ${ }^{2}$ A. Vallecchi, ${ }^{3}$ M. Albani, ${ }^{3}$ A. Le Beulze,${ }^{4}$ S. Gomez-Grana, ${ }^{4}$ \\ E. Duguet, ${ }^{4} \mathrm{~S}$. Mornet, ${ }^{4}$ and M. Treguer-Delapierre ${ }^{4}$ \\ ${ }^{1}$ Université Bordeaux, CNRS, CRPP-UPR 8641, 115 Avenue Albert Schweitzer, 33600 Pessac, France \\ ${ }^{2}$ Université Bordeaux, CNRS, Solvay, LOF-UMR 5258, 178 Avenue Albert Schweitzer, 33600 Pessac, France \\ ${ }^{3}$ Department of Information Engineering, University of Siena, Via Roma 56, 53100 Siena, Italy \\ ${ }^{4}$ Université Bordeaux, CNRS, ICMCB-UPR 9048, 87 Avenue Albert Schweitzer, 33600 Pessac, France \\ (Received 24 August 2015; revised manuscript received 1 December 2015; published 18 December 2015)
}

\begin{abstract}
We present a full experimental characterization of isotropic artificial optical magnetism at visible frequencies produced by "raspberrylike" plasmonic nanoclusters synthesized by a bottom-up approach. Experimental measurements of extinction and light scattering associated with numerical simulations enable one to extract absolute values of the magnetic scattering cross section across the whole visible spectral range. The strength of the resonant magnetic response of a single magnetic nanocluster is illustrated as the effective magnetic permeability of a homogeneous sphere of identical volume.
\end{abstract}

DOI: 10.1103/PhysRevB.92.220414

PACS number(s): 78.67.Bf, 75.75.-c, 42.68.Mj, 78.20.Ci

Introduction. The ability to control simultaneously the dielectric permittivity $\varepsilon(\lambda)$ and the magnetic permeability $\mu(\lambda)$ of a material in each point of space and for visible wavelengths is key to accessing unprecedented control of light propagation, and, further, to allowing applications such as cloaking, light harvesting, optical design, or optical analog computing [1,2]. While metal-dielectric composite materials give access to a wide range of positive and negative values of the real part of the permittivity in a broad frequency range, controlling the magnetic permeability is significantly more challenging. It is indeed well known that the optical response of natural materials illuminated by visible light is dominated by the polarization of electric charges rather than by the magnetization generated by currents [3]. The search for optical magnetism $(\mu \neq 1)$ in artificial materials has, however, become a key issue in the emerging field of metamaterials since the pioneering works of Veselago [4] and Pendry [5], who pointed out the unprecedented properties of perfect lensing and subwavelength imaging [6].

At microwave frequencies, the resonant circuit design of the so-called split ring resonator (SRR) has been successfully proposed by Pendry [5] and Smith [7], hence enabling the achievement of significantly resonant permeability and the demonstration of negative refraction. A magnetic response has been subsequently obtained at higher frequencies by the miniaturization of SRR-like arrays and multilayer fishnet structures [8]. Although quite successful in the production of optical magnetism up to visible light frequencies, such nanostructures fabricated by top-down lithography techniques are essentially two dimensional and highly anisotropic.

On the other hand, the concept of isotropic magnetic meta-atoms has been proposed by Simovski and Tretyakov [9] as magnetic nanoclusters (MNCs) made of a core dielectric nanoparticle surrounded by metallic nanosized satellites. The magnetic polarization is produced by rings of plasmonic currents $[10,11]$ whereas the uniform distribution of satellites around the core ("raspberry" model) warrants the isotropic response. Numerical simulations indeed confirm that a significant optical magnetism is expected [12], even in the presence of structural disorder which may result from bottom-up fabrication techniques [13]. The synthesis of MNCs based on the Simovski-Tretyakov design was first reported in 2011 by Mühlig et al. [14], who self-assembled gold satellites onto core silica nanoparticles by electrostatic interactions. These authors showed that the extinction measurements were consistent with the presence of a magnetic mode. More recently, Sheikoleslami et al. [15] reported on the synthesis and optical characterization of MNCs made of a polystyrene core coated with silver spheres. An elegant experiment of static light scattering was used to distinguish the magnetic scattering from the electric one at a wavelength of $644 \mathrm{~nm}$. A high magnetic to electric scattering ratio of $12 \%$ was hence measured. However, this measurement was performed at a single wavelength that may not correspond to the maximum optical magnetism. Qian et al. [16] used a similar light scattering technique to reveal the magnetic/multipolar response of multilayer aggregates of plasmonic nanoparticles around a dielectric core, different from the Simovski-Tretyakov design.

The aim of the present Rapid Communication is to provide a complete experimental optical study of the MNCs by combining spectrophotometry, static light scattering (SLS), measuring separately the magnetic and electric scattering, including multipole contributions, and dynamic light scattering (DLS) assessing the size of the scatterers. A full interpretation of the SLS experiments is provided by numerical simulations. The main result is an experimentally quantitative determination of the magnetic scattering cross section across the whole visible spectrum, and evidence of a significant resonant isotropic optical magnetism.

Numerical simulations. The electromagnetic response of MNCs was computed numerically by Vallecchi et al. [12] using a model based on the single dipole approximation of the metallic satellites in conjunction with a multipole expansion of the cluster. Following Ref. [12], we started the numerical approach with the construction of the MNCs. The arrangement of spherical nonoverlapping satellites onto the surface of a core sphere derives from the topological problem of distributing nonintersecting circles on the surface of a sphere $[17,18]$. The final state is obtained by defining an effective repulsion energy that is subsequently minimized to evenly distribute the satellites around the core sphere by maximizing the interparticle distance between satellites. Real MNCs may 


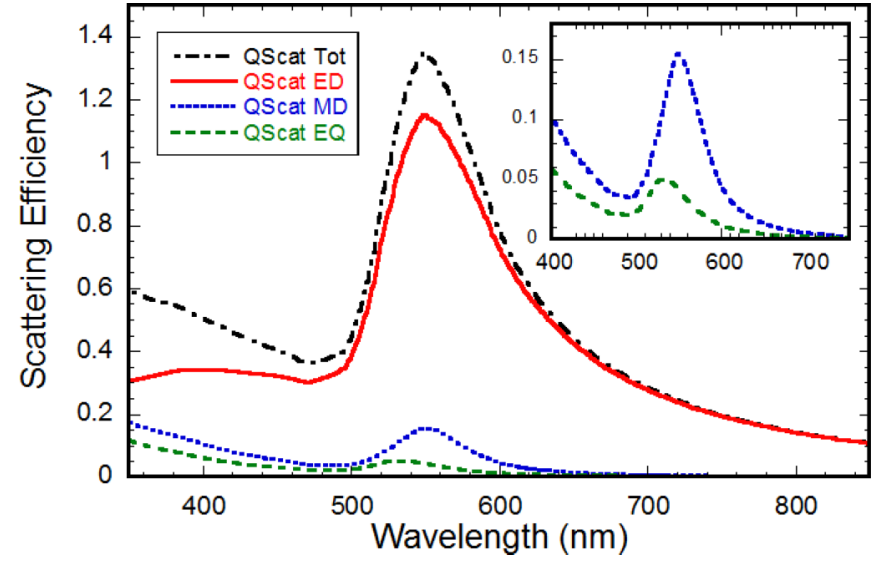

FIG. 1. (Color online) Numerical simulation of the scattering efficiencies for a nanocluster made of a silica core of diameter 106 $\mathrm{nm}$ surrounded by 26 gold satellites of diameter $30 \mathrm{~nm}$. The electric dipolar (ED), magnetic dipolar (MD), and electric quadrupolar (EQ) contributions are shown. The inset shows a magnification of the MD and EQ efficiencies.

exhibit more irregular distributions of the satellites around the core. Vallecchi et al. showed that the optical properties of the MNCs are weakly affected by the presence of positional irregularities [13].

In a second step, we employed a $T$-matrix code developed by Mackowski [19] to simulate the electromagnetic response of the MNCs. It is a FORTRAN code providing the solution to Maxwell's equations for an ensemble of nonoverlapping spheres. Following the formalism of the Mie theory describing the scattering of a single sphere [20], the elements of the scattering matrix are expanded in a series of scattering coefficients $a_{i}^{\mathrm{MNC}}$ and $b_{i}^{\mathrm{MNC}}$ computed for the whole cluster. The first-order coefficients $a_{1}^{\mathrm{MNC}}$ and $b_{1}^{\mathrm{MNC}}$ correspond respectively to the electric (ED) and magnetic (MD) dipolar modes of the $\mathrm{MNC}, a_{2}^{\mathrm{MNC}}$ to the electric quadrupole radiation (EQ), and so on. The general result is illustrated in Fig. 1. The simulated efficiencies of the absorption and scattering modes are computed for a MNC made of a silica core of diameter 106 $\mathrm{nm}$ surrounded by 30 gold satellites of diameter $30 \mathrm{~nm}$ in a water ambient medium $(\varepsilon=1.77)$. All modes display a strong resonance in the plasmon band $(530-560 \mathrm{~nm})$. The scattering efficiency of the magnetic dipole is about six times lower than the electric mode and 3.7 times higher than the EQ mode which dominates the series of multipolar modes. We checked that varying the direction of polarization or propagation of the incident electromagnetic wave produces no noticeable variation, as expected for isotropic scatterers.

Optical measurements. The MNCs were synthesized by electrostatic self-assembly of silica particles of diameter 106 $\pm 3 \mathrm{~nm}$ and gold satellites of diameter $30 \pm 2 \mathrm{~nm}$. Details of the synthesis will be described elsewhere [21]. In brief, the positive charge of the silica cores was obtained by coating them with a cationic polyelectrolyte, whereas the gold satellites were overall negatively charged at the output of synthesis [22]. A fine tuning of the ratio of the concentration of cores and satellites during the self-assembly process yields a narrow distribution of MNC clusters. A transmission electron

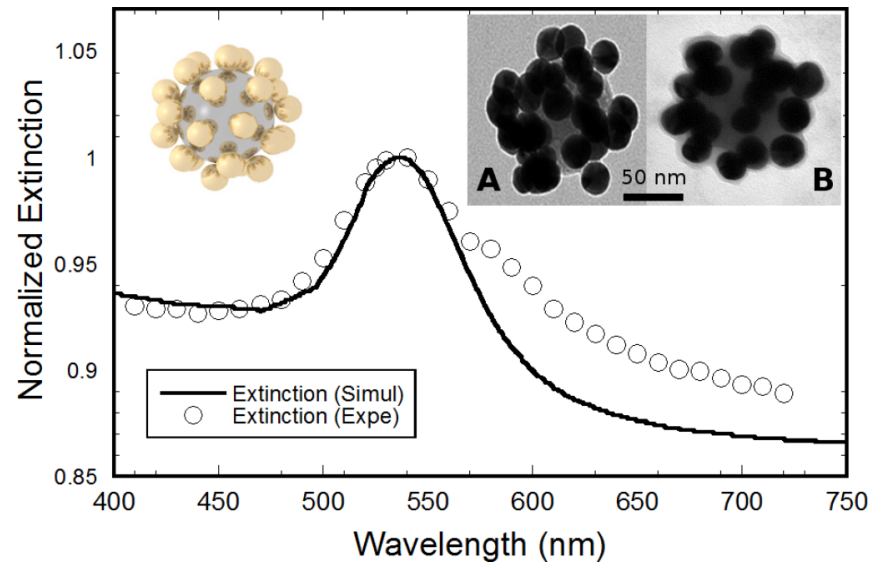

FIG. 2. (Color online) Normalized absorbance of a suspension of MNCs (circles). The left inset shows the theoretical model of an isotropic magnetic nanocluster (MNC) by Simovski and Tretyakov. The solid line is a numerical simulation for 26 satellites. The right inset shows a TEM micrograph of MNCs used in the present study (A). The core is a silica NP of diameter $106 \mathrm{~nm}$, and the satellites are gold NPs of diameter $30 \mathrm{~nm}$. The MNC in (B) was encapsulated in a thin silica layer to minimize perturbations of the distribution of satellites on the TEM grid.

microscopy (TEM) micrograph of the as-obtained MNCs is shown in the inset of Fig. 2. Figure 2 shows the absorbance spectrum of the suspension of MNCs. A plasmon resonance is observed with a maximum centered at $540 \mathrm{~nm}$, in good agreement with numerical simulations despite a slower than expected decay at long wavelengths which we assign to the presence of aggregates, as discussed below.

The scattering properties of dilute suspensions of MNCs were studied across the whole visible spectrum by a polarization resolved light scattering setup described in Fig. 3. The scattering angle $\theta$ is set at $90^{\circ}$. The incident field $\boldsymbol{E}_{\boldsymbol{i}}$ propagating along $z$ is linearly polarized in the $(x, y)$ plane at angle $\varphi-\pi / 2$ from the scattering plane $(y, z)$. We use the amplitude scattering matrix formalism [20]

$$
\left(\begin{array}{c}
E_{\| S} \\
E_{\perp S}
\end{array}\right)=\frac{e^{i k r}}{-i k r}\left(\begin{array}{ll}
S_{2} & S_{4} \\
S_{3} & S_{1}
\end{array}\right)\left(\begin{array}{c}
E_{i} \cos \varphi \\
E_{i} \sin \varphi
\end{array}\right) .
$$

$E_{\| S}$ and $E_{\perp S}$ are the components of the scattered field parallel and perpendicular to the scattering plane. The case of an isotropic scatterer has been worked out by Sharma [23]: The

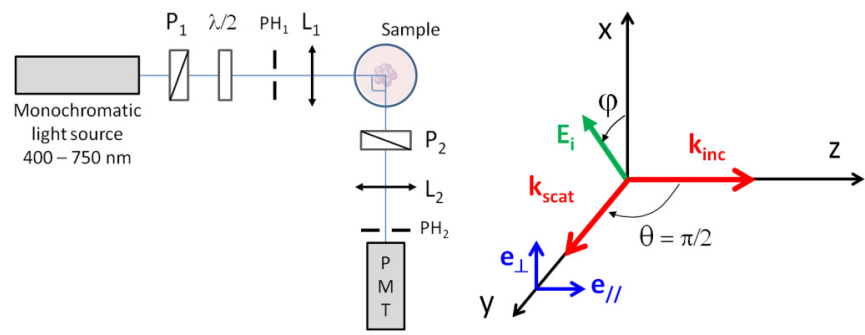

FIG. 3. (Color online) Schematic view of the light scattering setup (left) and scattering geometry (right). The output polarizer P2 enables the collection of scattering along the transverse $\left(\mathbf{e}_{\perp}\right)$ and axial $\left(\mathbf{e}_{\|}\right)$directions. 
$S_{i}$ coefficients do not depend on $\varphi$ and the nondiagonal terms $S_{3}$ and $S_{4}$ vanish. Moreover, at a scattering angle $\theta=90^{\circ}$, the scattering amplitude $S_{1}$ contains the radiation of the electric dipole only, whereas $S_{2}$ cumulates the radiations of the magnetic dipole and the electric quadrupole [23].

The intensities measured along the two output polarizations for isotropic scatterers then read

$$
\begin{aligned}
I_{\perp S}(\varphi)= & I_{0}(\lambda) \frac{N_{\mathrm{MNC}}}{k^{2}}\left|S_{1}\left(\theta=90^{\circ}\right)\right|^{2} \\
& \delta \Omega T(\lambda) g(\lambda, \delta \Omega) \sin ^{2} \varphi=A_{\perp}(\lambda) \sin ^{2} \varphi \\
I_{\| S}(\varphi)= & I_{0}(\lambda) \frac{N_{\mathrm{MNC}}}{k^{2}}\left|S_{2}\left(\theta=90^{\circ}\right)\right|^{2} \\
& \delta \Omega T(\lambda) g(\lambda, \delta \Omega) \cos ^{2} \varphi=A_{\|}(\lambda) \cos ^{2} \varphi,
\end{aligned}
$$

in which $I_{0}(\lambda)$ is the spectral irradiance of the incident beam, $N_{\text {MNC }}$ is the number of MNC scatterers in the scattering volume, and $\delta \Omega$ is the solid angle of the detection window. $g(\lambda, \delta \Omega)$ is an unknown function that accounts for the spectral sensitivity of the detector and optical transmission or reflection of all optical elements. The spectral transmission $T(\lambda)$ accounts for the absorbance of the suspension.

Experimentally, a supercontinuum white source (SuperK EXB-6 with SuperK Varia monochromator from NKT Photonics) was used to deliver a monochromatic light beam. The adjustable bandwidth was set to $6 \mathrm{~nm}$. The linear polarization of the incident light was set by a Glan-Taylor polarizer (Thorlabs GL5-A). An achromatic zero-order half-wave plate (Newport 10RP52-4) mounted on a rotation stage was used to rotate the incident polarization by an angle $\varphi$. The scattered light was collected with a photomultiplier tube (Brookhaven Instrument) at a fixed scattering angle $\theta=90^{\circ}$. The polarization of the scattered light was analyzed by a Glan-Taylor polarizer set perpendicular or parallel to the scattering plane to collect the $I_{\perp S}$ and $I_{\| S}$ signals, respectively.

The $I_{\perp S}$ and $I_{\| S}$ signals are shown in the inset of Fig. 4 versus the direction $\varphi$ of the incident polarization at $\lambda=$ $550 \mathrm{~nm}$. They are fitted for each wavelength to oscillating functions $B_{\perp}+A_{\perp} \cos ^{2} \varphi$ and $B_{\|}+A_{\|} \sin ^{2} \varphi$, respectively. The common background $B_{\perp} \cong B_{\|}$is less than $4 \%$ of the amplitude of the ED mode, hence confirming the isotropic response of the MNCs [23].

The ratio $A_{\|}(\lambda) / A_{\perp}(\lambda)$ measuring unambiguously the ratio of the MD and EQ contributions to the ED scattering is shown in Fig. 4, together with the simulated value $\left|S_{2}\right|^{2} /\left|S_{1}\right|^{2}$ computed for several numbers of satellites. The experimental data exhibit a clear maximum of $4.5 \%$ at $540 \mathrm{~nm}$, which is well reproduced by a distribution of 26 gold satellites around the core. This number is consistent with TEM views of the MNC (Fig. 2).

The MD+EQ and ED contributions can be extracted separately by normalizing the data with a reference dispersion of calibrated silica nanoparticles $\left(D_{\mathrm{SiO}_{2}}=106 \mathrm{~nm}\right)$ in water. The scattered intensity from the reference sample is similar to Eq. (2a) by replacing $\left|S_{1}\left(\theta=90^{\circ}\right)\right|^{2} / k^{2}$ with $\sigma_{\text {ref }}^{\left(\theta=\varphi=90^{\circ}\right)}$, the differential scattering cross section of a silica particle in water at scattering angles $\theta=\varphi=90^{\circ}$. The MNC and reference signals [24] were collected in the same experimental conditions so that the quantities $I_{0}, \delta \Omega$, and $g(\lambda, \delta \Omega)$ are the

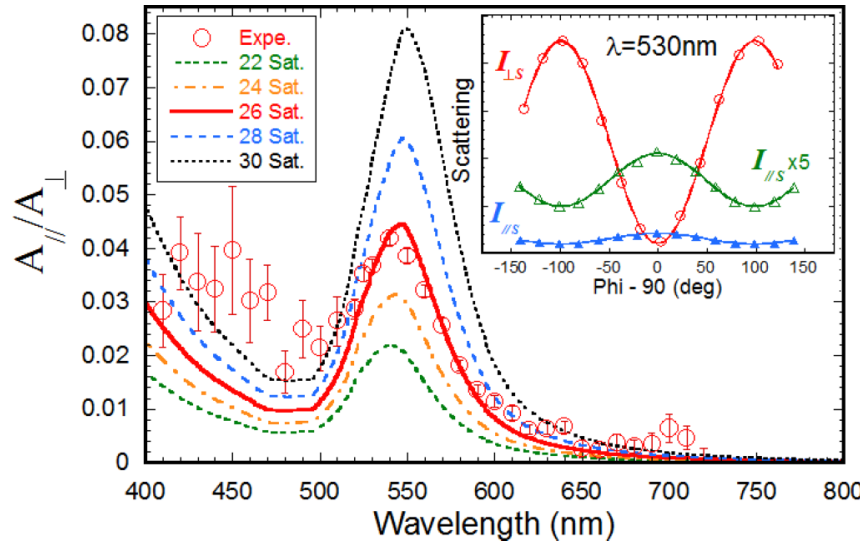

FIG. 4. (Color online) Ratio of axial to transverse scattering $A_{H} / A_{V}$ vs wavelength (red circles). The lines are numerical simulations vs the number of satellites. The best fit is obtained for 26 satellites. The large error bars at short wavelengths are due to a noisy scattering signal and to some deviation of the broadband retardation plate from the exact half wave. The inset shows transverse (red circles) and axial (blue triangles) scattering measured at $\lambda=530 \mathrm{~nm}$. The solid lines are fits to the $\sin ^{2}$ and $\cos ^{2}$ functions, respectively.

same for MNCs and for reference. Dividing Eqs. (2) for MNCs by the reference yields the differential scattering cross sections of a MNC along the particular directions $(\theta, \varphi)$ :

$$
\begin{aligned}
\sigma_{\perp}^{\mathrm{ED}}\left(\theta=\varphi=90^{\circ}\right) & =\frac{\left|S_{1}\left(\theta=90^{\circ}\right)\right|^{2}}{k^{2}} \\
& =K \frac{A_{\perp}(\lambda)}{A_{\perp}^{\mathrm{ref}}(\lambda)} \frac{T_{\mathrm{ref}}(\lambda)}{T(\lambda)} \sigma_{\mathrm{ref}}^{\theta=\varphi=90^{\circ}}(\lambda), \\
\sigma_{\|}^{\mathrm{MD}+\mathrm{EQ}}\left(\theta=90^{\circ}, \varphi=0\right) & =\frac{\left|S_{2}\left(\theta=90^{\circ}\right)\right|^{2}}{k^{2}} \\
& =K \frac{A_{\|}(\lambda)}{A_{\perp}^{\mathrm{ref}}(\lambda)} \frac{T_{\mathrm{ref}}(\lambda)}{T(\lambda)} \sigma_{\mathrm{ref}}^{\theta=\varphi=90^{\circ}}(\lambda) .
\end{aligned}
$$

Superscripts ED and MD+EQ remind that $\sigma_{\perp}$ and $\sigma_{\|}$are respectively dominated by the scattering of the electric dipole and of the added contribution of the magnetic dipole and electric quadrupole. $K=N_{\text {ref }} / N_{\mathrm{MNC}}$ is a constant independent of wavelength.

The scattering cross section of a silica sphere in water in transverse polarization $\sigma_{\text {ref }}^{\theta=\varphi=90^{\circ}}$ is calculated by the Mie theory $[20,25]$. The transmissions of highly dilute suspensions are dominated by the absorbance of water so that the ratio $T_{\text {ref }}(\lambda) / T(\lambda)$ is simply 1 . Due to a large uncertainty in the concentration of the MNCs after synthesis and purification, the constant $K$ could not be determined accurately.

The scattering cross sections are shown in Fig. 5. The transverse signal $K A_{\perp} / A_{\perp}^{\text {ref }} \sigma_{\text {ref }}^{\theta=\varphi=90^{\circ}}$ exhibits a broad maximum at $\lambda=580 \mathrm{~nm}$, consistent with a classical localized plasmon resonance. The spectral response of the axial signal $K A_{\|} / A_{\perp}^{\text {ref }} \sigma_{\text {ref }}^{\theta=\varphi=90^{\circ}}$ exhibits a sharper maximum at $\lambda=$ $550 \mathrm{~nm}$. Numerical simulations of $\left|S_{1}\right|^{2} / k^{2}$ and $\left|S_{2}\right|^{2} / k^{2}$ taking 26 satellites are displayed on the same graph. The 


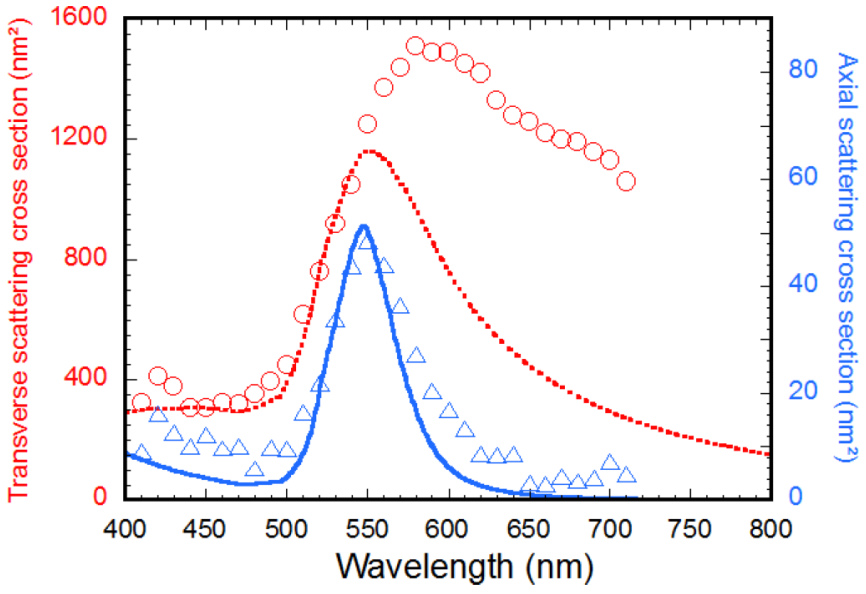

FIG. 5. (Color online) Transverse (red circles/dashed line) and axial (blue triangles/solid line) differential scattering cross sections measured at $\theta=\pi / 2$. The symbols denote experimental data obtained after normalization by a reference suspension of silica NPs. The lines show numerical simulations $\left[\left|S_{1}(\theta=\pi / 2, \varphi=0)\right|^{2} / k^{2}\right.$ and $\left|S_{2}(\theta=\varphi=\pi / 2)\right|^{2} / k^{2}$, respectively] for a MNC with 26 satellites.

unknown constant $K=N_{\text {ref }} / N_{\text {MNC }}$ is set to 110 to optimize the agreement between experiment and simulation for axial scattering. The position and width of the axial signal are hence well reproduced. The transverse scattering matches the simulation at short wavelengths. It displays, however, a significant additional contribution at wavelengths larger than $550 \mathrm{~nm}$. A similar effect is also visible on the axial signal, but the difference between the experimental and simulated signals above $550 \mathrm{~nm}$ is much weaker.

In order to understand the origin of this additional longwavelength contribution, the sizes of the MNCs and of the free satellites were assessed by dynamic light scattering. Suspensions were illuminated by a solid state continuous laser beam (Spectra-Physics, $\lambda=644 \mathrm{~nm}$ ). The time correlation function of the scattered signal was recorded at several angles with a Brookhaven BI-9000AT autocorrelator. Numerical inversion of the autocorrelation functions, using a regularized Laplace inversion (Contin type of fit), provided the radius distribution of the scatterers. Results for MNCs reveal a broad and asymmetric distribution extending from 90 to $200 \mathrm{~nm}$ with a maximum around $140 \mathrm{~nm}$, together with a weaker peak centered around $18 \mathrm{~nm}$, which can be assigned to the presence of free satellites in the suspension. The main population reveals the presence of nano-objects significantly larger than the MNCs (of nominal radius $=83 \pm 5 \mathrm{~nm}$ ). We assign this population to aggregates of free satellites as well as to possible aggregates of MNCs which could not be separated by the purification process. Aggregates of gold nanoparticles (NPs) are known to generate a redshifted plasmon resonance [26,27]. This is consistent with our observation of an additional signal at wavelengths longer than the main plasmon peak in the extinction curve (Fig. 2) and in the ED scattering curve (Fig. 5). An important result is that the axial scattering appears to be weakly affected by the presence of bigger objects which do not contribute much to the MD and EQ responses.
Finally, we deduce the MD and EQ contributions separately from numerical simulations. The best adjustment obtained for 26 satellites yields the maxima of the MD and EQ scattering efficiencies of 0.16 and 0.05 , respectively (Fig. 1). In order to evaluate the strength of the observed magnetic response, we estimate the effective magnetic permeability $\mu_{\mathrm{eff}}^{\mathrm{MNC}}$ of a fictional homogeneous sphere of equivalent volume $V$ which would produce the same dipolar scattering as a MNC. The effective magnetic polarizability of a MNC defined as $\mathbf{m}=$ $\beta_{\mathrm{eff}}^{\mathrm{MNC}} \mathbf{H}$ reads [20,28]

$$
\beta_{\mathrm{eff}}^{\mathrm{MNC}}=i \frac{4 \pi}{k^{3}} S_{2}^{\mathrm{dipole}}=3 V \frac{\mu_{\mathrm{eff}}^{\mathrm{MNC}}-1}{\mu_{\mathrm{eff}}^{\mathrm{MNC}}+2},
$$

in which $S_{2}^{\text {dipole }}=3 / 2 b_{1}^{\mathrm{MNC}}$ is the dipolar contribution to the $S_{2}$ element of the scattering matrix. The effective permeability of the equivalent sphere, shown in Fig. 6, exhibits a sharp magnetic resonance centered at $552 \mathrm{~nm}$. The real part displays a significant variation from 0.9 to 1.6 .

The effective permeability $\mu_{\mathrm{eff}}^{\text {material }}$ of a bulk material made of a dispersion of such MNCs with a volume fraction $f$ can be derived by a generalization of the Maxwell-Garnett mixing rule to magnetic dipoles [10,29],

$$
\frac{\mu_{\mathrm{eff}}^{\text {material }}-1}{\mu_{\mathrm{eff}}^{\text {material }}+2}=f \frac{\mu_{\mathrm{eff}}^{\mathrm{MNC}}-1}{\mu_{\mathrm{eff}}^{\mathrm{MNC}}+2} .
$$

Equation (5), which ignores coupling of the neighboring MNCs, is expected to be valid at low volume fractions only. Nonetheless, the Maxwell-Garnett rule is often used to provide an upper estimate of the resonance in a dense material. Setting, for instance, the maximum compactness of a random packing of hard spheres $f=64 \%$ in Eq. (5) yields a magnetic permeability ranging from 0.95 to 1.36 (dashed line in Fig. 6).

This work demonstrates that the "raspberrylike" model of magnetic nanoclusters proposed by Simovski and Tretyakov generates the expected isotropic magnetic response. We showed that spectroscopic polarized light scattering combined with numerical simulations enables a full characterization of this magnetic response, which is unambiguously linked to the plasmon resonance of the metallic satellites. Normalization

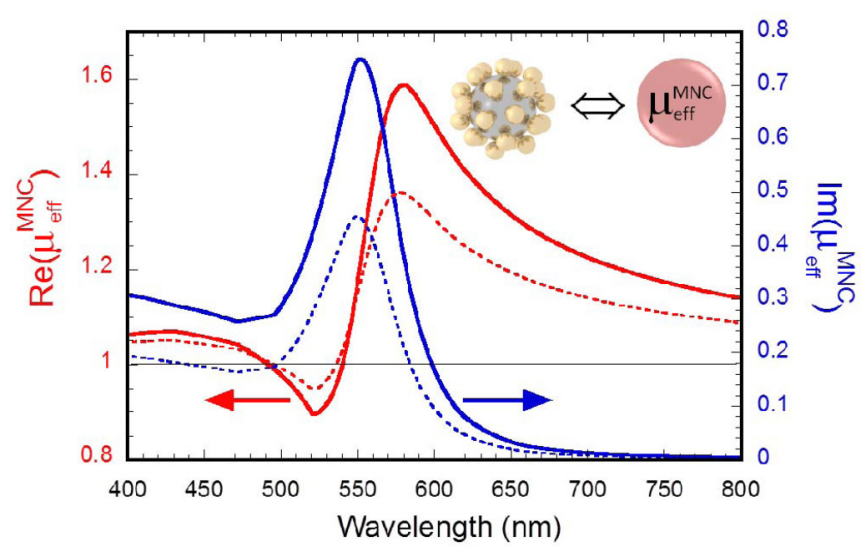

FIG. 6. (Color online) Effective magnetic permeability of a homogeneous sphere equivalent to a MNC (solid line). The dashed line shows the magnetic permeability computed for a bulk material made by random packing of such MNCs without coupling. 
of experimental data with a reference suspension of silica nanoparticles makes it possible to obtain separately the electric and magnetic scattering cross section of the nanoclusters. The full validation of the Simovski-Tretyakov model opens the way to the fabrication of bulk isotropic materials exhibiting a relative magnetic permeability significantly different from 1 , hence breaking the classical Landau-Lifshitz rule for visible light. Applications to impedance matching (high $\mu$ ) or negative index (negative $\mu$ ) clearly require a more intense magnetic response. Increasing the number of satellites, exchanging gold for silver [15], and changing the shapes of the satellites are current directions of research.

This work was supported by the LabEx AMADEus (ANR10-LABX-42) in the framework of IdEx Bordeaux (ANR-10IDEX-03-02), France.
[1] J. B. Pendry, D. Schurig, and D. R. Smith, Science 312, 1780 (2006).

[2] A. Silva, F. Monticone, G. Castaldi, V. Galdi, A. Alù, and N. Engheta, Science 343, 160 (2014).

[3] See, for instance, L. D. Landau and E. M. Lifshitz, Electrodynamics of Continuous Media (Pergamon, New York, 1960).

[4] V. G. Veselago, Sov. Phys. Usp. 10, 509 (1968).

[5] J. B. Pendry, A. J. Holden, D. J. Robbins, and W. J. Stewart, IEEE Trans. Microwave Theory Tech. 47, 2075 (1999).

[6] J. B. Pendry, Phys. Rev. Lett. 85, 3966 (2000).

[7] D. R. Smith, Willie J. Padilla, D. C. Vier, S. C. Nemat-Nasser, and S. Schultz, Phys. Rev. Lett. 84, 4184 (2000).

[8] A review of the race towards higher frequencies can be found in C. M. Soukoulis and M. Wegener, Nat. Photonics 5, 523 (2011).

[9] C. R. Simovski and S. A. Tretyakov, Phys. Rev. B 79, 045111 (2009).

[10] A. Alù, A. Salandrino, and N. Engheta, Opt. Express 14, 1557 (2006).

[11] A. Alù and N. Engheta, Phys. Rev. B 78, 085112 (2008).

[12] A. Vallecchi, M. Albani, and F. Capolino, Opt. Express 19, 2754 (2011).

[13] A. Vallecchi, M. Albani, and F. Capolino, Opt. Express 21, 7667 (2013).

[14] S. Mühlig, A. Cunningham, S. Scheeler, C. Pacholski, T. Bürgi, C. Rockstuhl, and F. Lederer, ACS Nano 5, 6586 (2011).

[15] S. N. Sheikholeslami, H. Alaeian, A. L. Koh, and J. A. Dionne, Nano Lett. 13, 4137 (2013).

[16] Z. Qian, S. P. Hastings, C. Li, B. Edward, C. K. McGinn, N. Engheta, Z. Fakhraai, and S.-J. Park, ACS Nano 9, 1263 (2015).
[17] B. W. Clare and D. L. Kepert, Proc. R. Soc. London, Ser. A 405, 329 (1980).

[18] D. A. Kottwitz, Acta Crystallogr., Sect. A 47, 158 (1991).

[19] http://www.eng.auburn.edu/users/dmckwski/scatcodes/

[20] C. F. Bohren and D. R. Huffman, Absorption and Scattering of Light by Small Particles (Wiley-Interscience, New York, 1983).

[21] A. Le Beulze, S. Gomez-Grana, H. Gehan, S. Mornet, S. Ravaine, M. Correa-Duarte, L. Guerrini, R. A. Alvarez-Puebla, E. Duguet, and M. Treguer-Delapierre (unpublished).

[22] N. G. Bastús, J. Comenge, and V. Puntes, Langmuir 27, 11098 (2011)

[23] N. L. Sharma, Phys. Rev. Lett. 98, 217402 (2007).

[24] See Supplemental Material at http://link.aps.org/supplemental/ 10.1103/PhysRevB.92.220414 for a plot of the reference signal measured on silica nanoparticles.

[25] M. Daimon and A. Masumura, Appl. Opt. 46, 3811 (2007); I. H. Malitson, J. Opt. Soc. Am. 55, 1205 (1965).

[26] S. K. Ghosh and T. Pal, Chem. Rev. 107, 4797 (2013).

[27] N. G. Khlebtsov, V. A. Bogatyrev, A. G. Melnikov, L. A. Dykman, B. N. Khlebtsov, and Ya. M. Krasnov, J. Quant. Spectrosc. Radiat. Transfer 89, 133 (2004).

[28] A. García-Etxarri, R. Gómez-Medina, L. S. Froufz-Pérez, C. López, L. Chantada, F. Scheffold, J. Aizpurua, M Nieto-Vesperinas, and J. J. Sáenz, Opt. Express 19, 4815 (2011).

[29] A. H. Sihvola and I. V. Lindell, in Dielectric Properties of Heterogeneous Materials, edited by A. Priou, Progress in Electromagnetics Research Vol. 6 (Elsevier, New York, 1992), Chap. 4, pp. 153-180. 